\title{
MODELOS DE COMPRAS PÚBLICAS PARA CONSUMO SOCIAL EN ESTABLECIMIENTOS EDUCACIONALES
}

\author{
Public procurement schemes for social consumption at educational institutions
}

\author{
Cindy Encina Galaz \\ Ingeniero Agrónomo por la Universidad de Chile. Santiago, Chile. cindy.encina@gmail.com \\ Sofía Boza Martínez \\ Ph.D. en Economía. Universidad de Chile. Santiago, Chile sofiaboza@u.uchile.cl
}

Marcos Mora González

Ph.D. en Economía de la Empresa Agroalimentaria. Universidad de Chile. Santiago, Chile mmorag@uchile.cl

\begin{abstract}
Cómo citar / How to cite
Encina, C., Boza, S. y Mora, M. (2015). Modelos de compras públicas para consumo social en establecimientos educacionales. Revista CEA, 1(1), 51-63.
\end{abstract}

Recibido: 6 de mayo de 2014

Aceptado: 17 de junio de 2014

\section{Resumen}

El escaso desarrollo rural y la falta de oportunidades que potencien el entorno local conllevan a una fuerte migración hacia las ciudades y al empobrecimiento de las zonas menos urbanizadas. Internacionalmente, existen casos donde los Programas de Alimentación Escolar (PAE) colaboran con las economías locales mediante la compra de alimentos a pequeños productores. En este contexto, el objetivo principal de este trabajo es definir las fortalezas y puntos críticos, para la implementación de un modelo de compras públicas de alimentos provenientes de la pequeña agricultura, para su consumo en establecimientos educacionales públicos de Chile. Para ello, además de la recopilación de información secundaria referente al modelo implantado en Brasil y funcionamiento del PAE chileno, se realizaron un total de 64 entrevistas a productores (30), apoderados (31) y responsables de comedores escolares (3) ubicados en la provincia de Colchagua (VI Región). Los resultados obtenidos mostraron una amplia aceptación de la participación de pequeños productores en el PAE chileno por parte de los entrevistados. No obstante, se identificaron algunas exigencias al modelo, como la regularidad de las entregas y calidad de los alimentos, la oportunidad en los pagos y el control y fiscalización del correcto funcionamiento de la cadena de suministro. Finalmente, la información recopilada fue sintetizada mediante una matriz FODA (Fortalezas, Oportunidades, Debilidades y Amenazas).

Palabras claves: Programas de Alimentación Escolar, agricultura familiar campesina, compras públicas.

\begin{abstract}
The limited rural development and the lack of opportunities to improve the local environment lead to a strong migration towards cities and to the impoverishment of the less urbanized areas. Internationally, there are cases where the School Feeding Programmes (SFPs) are collaborating with the local economies by buying their supplies from small scale producers. In this context, the main objective of this work is to define the strengths and critical points for the implementation of a scheme of public procurement of food from small farms for consumption in public educational institutions in Chile. In addition to collecting secondary information concerning the model implanted in Brazil and the operation of Chilean SFPs, a total of 64 interviews to producers (30), parents (31) and responsible for school canteens (3) placed in the province of Colchagua (O'Higgins Region) were done. The results showed a wide acceptance by respondents of the participation of small farmers in the Chilean SPF. However, some demands on the model were identified, as the timing of payments, regularity of deliveries and quality of the food offered, and the control and supervision of the proper functioning of the supply chain. Finally, the information gathered was synthesized using a SWOT matrix (strengths, weaknesses, opportunities and threats).
\end{abstract}

Keywords: School-Feeding Programs; Small-scale farming; Public procurements. 


\section{INTRODUCCIÓN}

La implementación de sistemas públicos para alimentación social constituye una innovadora política gubernamental para tratar de aumentar el bienestar, en especial cuando estos patrones de compras incluyen criterios como el apoyo a la agricultura familiar campesina (AFC) o factores medioambientales.

Un ejemplo de esto es Brasil, país pionero en Latinoamérica en la implementación de los modelos de compras públicas señalados, al adquirir alimentos provenientes de la agricultura familiar campesina para el consumo en escuelas estatales. Esta iniciativa se encuentra respaldada por el Artículo 14 de la nueva Ley 11.947 de 2009, el cual señala que del total de los recursos financieros transferidos por el Fondo Nacional para el desarrollo de la Educación (FNDE), en el ámbito del Programa Nacional de Alimentación Escolar (PNAE), al menos un $30 \%$ deberá ser utilizado en la adquisición de productos alimenticios directamente de la agricultura familiar y del empresario familiar rural o de sus organizaciones.

En Chile, la Junta Nacional de Auxilio Escolar y Becas (JUNAEB) es la encargada del funcionamiento del Programa de Alimentación Escolar (PAE) nacional en establecimientos municipales y particulares subvencionadas, cubriendo alrededor de un tercio de las necesidades nutricionales del día de los escolares de enseñanza básica y media, y entre un $45 \%$ y $50 \%$ en los preescolares (JUNAEB, 2014). Sin embargo, la compra de los alimentos utilizados en este programa se realiza mediante la selección y contrato de concesionarias, en los cuales no se incorporan criterios sociales 0 ambientales específicos como en Brasil.

Considerando la contextualización anterior, el objetivo del presente documento es definir las fortalezas, así como puntos críticos, para la implementación de un modelo de compras públicas de alimentos provenientes de la pequeña agricultura de estas características en establecimientos educacionales chilenos. Para ello, el trabajo realizado se estructura en los siguientes ejes: i) revisión de la experiencia brasileña, ii) exposición del funcionamiento del PAE en Chile, iii) presentación de los resultados de las entrevistas realizadas a informantes clave y iv) realización de un análisis FODA en base a la información levantada.

\section{MARCO CONCEPTUAL}

Las compras públicas son un mecanismo mediante el cual el Estado y sus administraciones pueden incidir en la economía. Según señala un estudio sobre compras públicas realizado por PNUMA (2012), a nivel general, el gasto público suele representar un $15-30 \%$ del PIB nacional y cada compra es una oportunidad de impulsar los mercados hacia la innovación y la sostenibilidad.

En este sentido, las iniciativas relativas a utilizar las compras públicas incorporando criterios sociales como una herramienta que incida en el desarrollo local han tomado importancia en el contexto internacional. Un ejemplo de esto es el funcionamiento de los PAE que obtienen parte de los alimentos desde la AFC.

En América Latina, la AFC otorga empleo aproximadamente a dos de cada tres agricultores, por lo que se podría afirmar que alrededor de 100 millones de personas dependen directamente de ella (BID y FAO, 2007). Igualmente, a nivel mundial, el $86 \%$ de los habitantes de zonas rurales tienen como principal fuente de ingreso la agricultura (Banco Mundial, 2008). Pese a su importancia relativa, el limitado acceso a mercados e inversión afecta la productividad y competitividad de la AFC, pudiendo encontrarse incluso los hogares dependientes en eventuales situaciones de 
vulnerabilidad. Lo anterior evidencia el rol central de la pequeña agricultura en las economías de los países latinoamericanos y la necesidad de políticas públicas en apoyo a la misma.

Por tanto, la participación de este sector productivo en iniciativas como los PAE sugiere una gran oportunidad para generar espacios para el desarrollo rural endógeno, mediante la circulación de los recursos en las zonas más sensibles económicamente. De hecho, según Figueroa et al. (2005), la descentralización ha demostrado crear condiciones para mejorar la calidad de los alimentos, reducir los costos, estimular la economía local, generar nuevos empleos, apoyar a los pequeños y medianos productores, así como fomentar la participación de los actores implicados para combatir la corrupción y desvíos de recursos.

Sin embargo, para lograr esta sinergia es necesaria la existencia de una agricultura familiar bien desarrollada que sea capaz de abastecer el sistema de alimentación. Según señala un estudio sobre alimentación escolar de FAO (2013), para establecer el enlace entre los PAE y la agricultura familiar se necesita de agricultores organizados $y$ capacitados, así como de un marco legal y normativo que facilite la participación de estos en las compras públicas del Estado. Esto, ya que según Márcia (2010), los procesos de licitación y las formalidades que estos modelos implican dificulta el acceso de la AFC a los mismos.

Finalmente, se necesita también de una comunidad participativa. Según CervatoMancuso et al. (2013), la calidad de la alimentación y nutricional en el ámbito de escolar es un compromiso que debe implicar la participación de nutricionistas, profesores, comunidad, gestores de las escuelas y de las entidades de gobierno.

\section{RESULTADOS Y DISCUSIÓN}

\section{Experiencia brasileña}

Desde hace años, uno de los ejes de la política brasileña ha sido garantizar la seguridad alimentaria de la población. Un ejemplo de esto es la iniciativa "Hambre Cero» iniciada el 2003, con el objetivo de erradicar el hambre y ayudar a las familias que aún presentan dificultades en el acceso a los alimentos básicos (FAO, 2006). Esta iniciativa está constituida por alrededor de 30 programas complementarios, dirigidos a combatir tanto las causas inmediatas como subyacentes del hambre y la inseguridad alimentaria. Dentro de esta línea se destacan: el Programa Nacional de Alimentación Escolar (PNAE), el Programa de Adquisición de Alimentos (PAA), que trabaja en conjunto con la Compañía Nacional de Abastecimiento (Conab), y el Programa Nacional de Fortalecimiento de la Agricultura Familiar (PRONAF), los cuales se analizarán a continuación, debido a su importancia en la conexión de la agricultura y la alimentación escolar.

\section{Programa Nacional de Alimentación Escolar (PNAE)}

Este programa se caracteriza por su universalidad (dirigido a todos los alumnos censados), equidad y la operación descentralizada, razones por las que se considera ejemplo en la gestión de los programas de alimentación escolar en el extranjero (Da Cunha et al., 2013). Tiene por objetivo contribuir con el crecimiento $y$ desarrollo biopsicosocial, el aprendizaje, el rendimiento escolar y la formación de hábitos saludables por parte de los estudiantes, a través de la educación alimentaria nutricional y del suministro de alimentos que cubran parte de las necesidades nutricionales durante el periodo lectivo (FNDE, 2012). 
Actualmente el PNAE se rige por la Ley 11.947 de 2009 , la cual establece que al menos un $30 \%$ de los recursos destinados por el FNDE se dedique a la obtención de alimentos provenientes de la agricultura familiar. La prioridad para la adquisición de estos alimentos son los productos generados en el mismo municipio, seguidos por la producción de agricultores de otros municipios rurales y finalmente de estados $u$ otras regiones del país.

Los recursos del programa provienen de la Tesorería Nacional, administradora de los fondos del gobierno federal. El FNDE se encarga de transferir los fondos a las entidades ejecutoras en 10 cuotas mensuales a partir del mes de febrero para la cobertura del año escolar. El uso de los recursos es monitoreado y supervisado directamente por los Consejos de Alimentación Escolar (CAE), FNDE, Tribunal de Cuentas de la Unión (TCU), Secretaría Federal de Control Interno (SFCl) y el Ministerio Público.

\section{Programa de Adquisición de Alimentos (PAA)}

Este programa fue creado en 2003 por el Gobierno Federal. Consiste básicamente en la adquisición, sin licitación, de productos provenientes de agricultura familiar a precios del mercado regional. Además, bajo la operación del Ministerio de Desarrollo Agrícola, proporciona recursos que permiten a las organizaciones de agricultores familiares crear un inventario de productos para su comercialización posterior, al mejor precio de mercado (IICA, 2007). Este programa se ejecuta en asociación con la Compañía Nacional de Abastecimiento (Conab), entidad encargada de mantener la regularidad del abastecimiento y la garantía de ingreso al productor rural en las compras, participando en la formulación y ejecución de políticas agrícolas de suministro (Conab, 2005).
Los alcances del PAA desde su implementación en 2003 hasta diciembre de 2010 corresponden a la compra de aproximadamente 2 millones de toneladas de alimentos. En 2008, 118.900 agricultores vendieron sus productos al Programa, siendo destinados a 16,8 millones de personas beneficiarias (MDA, 2012).

Por otro lado, con la finalidad de financiar los proyectos agrícolas de este sector y generar un mayor número de productos, el Programa Nacional de Fortalecimiento de la Agricultura Familiar (Pronaf), otorga varias líneas de financiamiento, dependiendo del público específico que se quiere atender, aplicando las tasas de interés más bajas que el mercado (MDA, 2014). En este sentido, desde 2003 se firmaron más de 10 millones de contratos y se entregaron 52.000 millones de reales en crédito agrícola, lo que corresponde al $85 \%$ del total de los recursos destinados al PRONAF desde su creación (Ibíd.)

\section{Situación actual en Chile}

Para lograr la comprensión del sistema de actuación pública que se lleva a cabo en Chile respecto a los Programas de Alimentación Escolar y al desarrollo de la Agricultura Familiar Campesina, se hace necesario analizar los principales organismos estatales involucrados en estas tareas. Por el lado de la distribución de las raciones alimenticias se tiene a la Junta Nacional de Auxilio Escolar y Becas (JUNAEB), encargada del Programa de Alimentación Escolar. Mientras que la agricultura familiar campesina se encuentra representada fuertemente por el Instituto de Desarrollo Agropecuario (INDAP). El detalle de estos programas se muestra a continuación. 
Junta Nacional de Auxilio Escolar y Becas (JUNAEB)

La JUNAEB es una institución del Estado, parte del Ministerio de Educación. Su misión es «liderar la implementación de políticas públicas, a través del diseño y gestión de productos, servicios y becas de calidad, destinados a la promoción de derechos y protección social de los estudiantes en Chile, en los niveles de enseñanza pre-básica, básica, media-secundaria y superior en condición o riesgo de vulnerabilidad, con las familias y la red pública y privada» (JUNAEB, 2008). Para esto JUNAEB coordina diferentes líneas de acción, especializándose en las siguientes tres áreas de apoyo social escolar: Programa de Salud Escolar (PSE), Programa de Becas y Programa de Alimentación Escolar (PAE). Dada la temática del presente artículo nos centraremos en la descripción de este último.

Programa de Alimentación Escolar (PAE). El Programa de Alimentación Escolar en Chile está a cargo de la Junta Nacional de Auxilio Escolar y Becas, quien administra los recursos asignados por el Estado para la ayuda de los escolares que se encuentran en riesgo biopsicosocial y acuden a establecimientos educativos municipales o particulares subvencionados.

Este programa tiene como fin último apoyar a los estudiantes combatiendo la deserción durante los 12 años de escolaridad. Para ello se entrega una alimentación complementaria y diferenciada, cubriendo parte de las necesidades nutricionales diarias de los estudiantes, a lo largo de todo el país, tanto de enseñanza media como básica, que se encuentran en situación de vulnerabilidad.

La ración alimenticia se traduce en un desayuno u «once» y almuerzo, según corresponda a las necesidades $y$ vulnerabilidad de los estudiantes.

Unidades involucradas. La unidad encargada de la coordinación y funcionamiento del
Programa de Alimentación Escolar es el Departamento de Alimentación Escolar, el cual, a su vez, cuenta con dos subunidades: la Unidad de Operaciones, responsable de definir, controlar y evaluar el presupuesto y su distribución; y la Unidad de Calidad, responsable de definir, controlar y evaluar los procesos relacionados con requisitos técnicos del programa.

Beneficiarios. La selección de los beneficiarios se realiza mediante el Sistema Nacional de Asignación con Equidad (SINAE), el cual utiliza una base de datos que contiene información proporcionada por los Ministerios de Desarrollo Social y de Educación, el Fondo Nacional de Salud (FONASA) y JUNAEB, del Registro de Estudiantes de Chile y su condición socioeconómica y de salud, además de información recopilada mediante la encuesta anual que realiza la JUNAEB a estudiantes. Esto permite identificar a los alumnos que requieren ayuda prioritaria.

Según cifras del Sistema Nacional de Asignación con Equidad (SINAE) para el 2010, el $64,11 \%$ (1.222.990) de los estudiantes de educación básica y el 66,64\% (617.160) de los estudiantes de educación media requerían de ayuda prioritaria.

Presupuesto. La ley de presupuestos de cada año indica los montos asignados a la institución JUNAEB para cada categoría. Para el año 2012 se contó con un presupuesto de 381.487.677 miles de pesos chilenos (US\$677.598.005), de los cuales 183.207.505 miles de pesos (US\$ 325.412.975) fueron asignados al programa de alimentación de enseñanza básica y 81.518 .051 miles de pesos (US\$144.792.275) para el programa de alimentación de enseñanza media (DIPRES, 2012); logrando la cobertura sobre 1.900 .000 raciones diarias.

Adquisición de alimentos. El servicio de alimentación es realizado por concesionarias que se encargan de la parte operativa del sistema, principalmente de la elaboración de 
los productos y su distribución a los diferentes establecimientos educacionales. JUNAEB, en tanto, define las bases técnicas donde se establecen las calidades de las materias primas y el producto final.

Para la contratación de las concesionarias se realizan licitaciones públicas donde estas compañías concursan. Las concesiones se adjudican cada tres años. El territorio nacional se divide en tres tercios y todos los años se licita uno. En cada licitación se evalúa el desempeño en la calidad de los servicios, en lo legal y en lo tributariocontable. El proceso se lleva a cabo mediante el portal de Mercado Público y no considera criterios sociales ni medioambientales.

Instituto de Desarrollo Agropecuario (INDAP)

El Instituto de Desarrollo Agropecuario, INDAP, es una institución pública dependiente del Ministerio de Agricultura. Se creó en 1962 con la misión de apoyar el desarrollo de los agricultores de pequeña escala mediante el fomento productivo. Actualmente, manifiesta como sus objetivos finales la superación de la pobreza, la sostenibilidad y la competitividad de la agricultura nacional.

En cuanto a los usuarios, clasifica como pequeño agricultor aquel que realiza la explotación de una superficie no mayor a 12 hectáreas de riego básico (HRB), una cantidad de activos no superior a las 3.500UF (US\$147.553) y que percibe ingresos provenientes principalmente de la actividad agrícola.

En cuanto a las funciones desempeñadas por INDAP se establecen tres formas de apoyo: asistencia técnica y capacitación de beneficiarios, asistencia crediticia directa y/o asistencia crediticia a las organizaciones de los beneficiarios (hasta el $95 \%$ del valor bruto del proyecto de inversión). Para lo anterior,
INDAP cuenta con múltiples programas de fomento, dentro de los cuales destaca el Programa de Desarrollo Local, encargado del apoyo productivo principalmente de los usuarios pertenecientes a la AFC.

\section{Programa de Desarrollo Local (PRODESAL).}

PRODESAL inicia en el año 1996 con el fin de apoyar la pequeña agricultura con un menor grado de desarrollo productivo, no más de 5 HRB (INDAP, 2008).

El programa es ejecutado fundamentalmente por los municipios seleccionados por INDAP mediante un concurso público. Estos eligen un equipo de trabajo integrado por profesionales y técnicos del ámbito silvoagropecuario, que presten servicio permanente a los usuarios (PUC, 2010).

El programa está dirigido a tres tipos de usuarios, según el objetivo y nivel productivo en el que se estén desenvolviendo (INDAP, 2010a). Estos corresponden a:

- Autoconsumo: usuarios cuyo principal destino de la producción es el autoconsumo familiar y subsistencia (sobre el 50\%).

- Transición: usuarios que se encuentran en proceso de superar la etapa de autoconsumo y subsistencia, produciendo, además del consumo familiar, una proporción de excedentes destinados a la venta.

- Comercial: usuarios para quienes el destino principal de su producción es realizar emprendimientos de negocios orientados a mercados más formales.

Características de los usuarios. En el estudio «Caracterización de usuarios Prodesal» publicado el año 2009 por INDAP, se muestra 
que para el año 2008 el $79 \%$ de los usuarios cuentan con la Ficha de Protección Social (FPS) utilizada por Chile Solidario, demostrando su vulnerabilidad. Por otra parte, el $60 \%$ de los mismos no posee activos rurales ni urbanos, lo cual es consistente con la característica de pertenecer a la población de menores recursos. En cuanto a su tipo de producción, el 59\% corresponde a agricultores de autoconsumo, mientras que el $41 \%$ se encuentran preferentemente vinculados al mercado.

Financiamiento y presupuesto. Las acciones del Programa son cofinanciadas por INDAP y los recursos aportados por las entidades ejecutoras. En este aspecto tenemos los recursos aportados por INDAP, más los otorgados por los municipios (10\% del aporte INDAP) dependiendo del tamaño y ubicación geográfica de la Unidad Operativa, teniendo en cuenta que las zonas aisladas, insulares o de condiciones especiales poseen un financiamiento superior para cubrir mayores costos de implementación (INDAP, 2010b).

\section{Entrevistas a informantes claves}

Considerando los datos recopilados, con el fin de obtener información de primera mano sobre la aceptabilidad de un modelo de compras públicas de alimentos a la AFC para la educación escolar en Chile, se realizó un total de 64 entrevistas a tres categorías de agentes que potencialmente estarían implicados en su implantación: a) pequeños productores (30), b) apoderados (31) y c) responsables de comedores escolares (3). Dichas encuestas se llevaron a cabo en la provincia de Colchagua, VI Región del Libertador Bernardo O’Higgins. La elección de esta zona sobre otras posibles se debe a que en ella podemos encontrar una importante cercanía geográfica entre productores agrícolas y consumidores.
Si bien el número de encuestas realizadas no corresponde a una muestra estadísticamente representativa, permite realizar un presondeo y sentar las bases de lo que podría ser un estudio más exhaustivo. En este contexto, a continuación, se exponen los principales resultados de entrevistas por segmento.

\section{Entrevistas a productores}

Mediante la información obtenida del Censo Agropecuario y Forestal del año 2007 se seleccionaron las comunas de la Provincia del Colchagua con mayor producción de hortalizas, debido a su importancia en la dieta escolar. Estas son: Chépica $(882,19$ ha.), Chimbarongo (1338,52 ha.) y Santa Cruz (577,15 ha.).

Los productores accedieron a contestar la encuesta de forma anónima. Los resultados más importantes se revelan a continuación.

Tipo de producción. Dentro de los entrevistados, el 33,33\% tenían más de un cultivo en su predio, mientras que el $46,67 \%$ presentaban solo un cultivo y el $13,33 \%$ se dedicaban a la producción de huevos. La superficie productiva promedio es de 2,15 ha., donde 12 agricultores presentan una superficie mayor a 1ha., 9 muestran una superficie menor a 1 ha y 5 poseen un tamaño aproximado de 1ha. Para los productores de huevos esta variable no se consideró.

Destino de la producción. En cuanto al destino de la producción, el $90 \%$ se dedica al comercio; el 3,33\% al autoconsumo y el $6,67 \%$ indicaron que su producción tiene doble propósito: autoconsumo y comercio (Figura 1). Dentro de los que comercializan el $76,67 \%$ lo hacen a ferias locales y de otras regiones, así como también a vecinos y/o de forma ambulante en la zona. Del resto, el 
$20 \%$ vende sus productos a instituciones (ej. Copeval, Coagra, Cinta Azul).

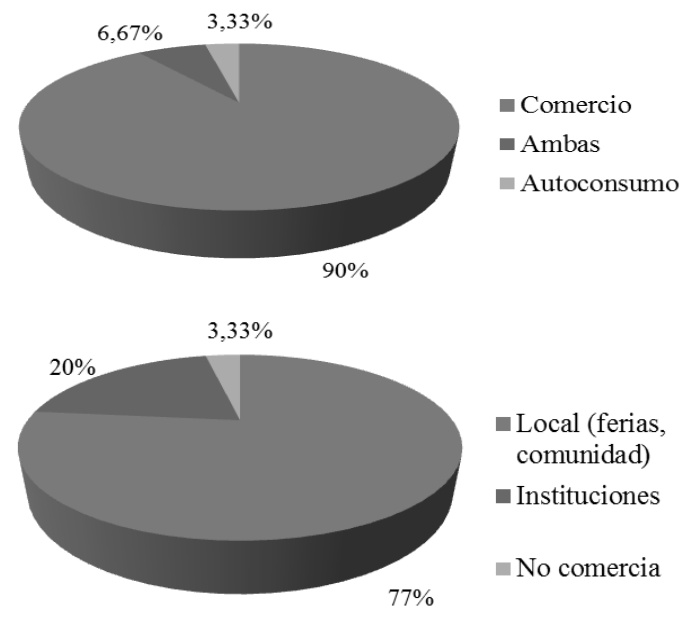

Figura 1. Destino de la producción

Figure 1. Production target

Implementación del Programa de Adquisición de Alimentos. Del total de los entrevistados, un $100 \%$ afirma que estaría dispuesto a participar del programa e invertir para mejorar la calidad, inocuidad y cantidad de su producción. Dentro de las herramientas necesarias para realizar esta inversión se mencionaron con gran frecuencia financiamiento y terreno (73,34\%).

Aspectos positivos y negativos. Dentro de los 30 entrevistados, los puntos positivos de la propuesta más señalados fueron: "contrato seguro y seriedad" (96,67\%); "mejora en los precios y aumentar ingresos» $(26,67 \%)$; «aumentar el nivel de producción» $(6,67 \%)$ y «disminuir el monopolio» $(3,33 \%)$. Solo el $30 \%$ percibió aspectos negativos, los temas señalados son: «incumplimiento de compra, plazos y pagos»; "rechazo de productos o un porcentaje alto de desecho» $y$ «el requisito de inicio de actividades».

Exigencias. Los requerimientos de los productores para participar en el Programa se agruparon en 5 categorías: forma de pago oportuna y en efectivo (73,33\%); seguridad de contrato serio y cumplimiento (40\%); precio y su mantención (20\%); y transparencia tanto del proceso como del control de calidad (3,33\%)

\section{Entrevistas a apoderados}

Se entrevistó a apoderados y encargados de cocina de 3 establecimientos educativos ubicados en las mismas comunas del origen de los productores en la muestra. A continuación los principales resultados de las entrevistas.

Identificación. La mayor parte de las familias de los apoderados entrevistados (35,48\%) tienen dos hijos; $25,8 \%$ tienen un hijo y otro $25,8 \%$ tienen tres hijos. El porcentaje restante se dividía entre familias de cuatro y cinco hijos. En cuanto a residencia, del total de entrevistados, el $55 \%$ declaró que la vivienda familiar se ubicaba en la zona urbana, mientras que el $45 \%$ restante reside en la zona rural.

Obtención de alimentos. En cuanto a la obtención de alimentos, el $64,52 \%$ de las respuestas se concentraron en supermercados y ferias conjuntamente, el porcentaje restante se divide en un mix que incluyen almacenes, verdulerías y supermercado conjuntamente. Ver Tabla 1.

Tabla 1. Obtención de los alimentos.

Table 1. Obtaining food

\begin{tabular}{lc}
\hline Origen & $\begin{array}{c}\text { Número de } \\
\text { familias }\end{array}$ \\
\hline Solo ferias & \\
Solo supermercados & 1 \\
Ferias y supermercados & 10 \\
Supermercados y & 9 \\
almacenes & 3 \\
Ferias, almacenes y & 7 \\
supermercados & 1 \\
Supermercados y otros & \\
(verdulería) & \\
\hline
\end{tabular}


Prioridad en la compra de alimentos. Para esta pregunta se indicaron cuatro parámetros (precio, calidad, origen y apariencia) a medir a la hora de comprar los alimentos. El análisis de los resultados arrojó que la variable considerada como más importante era el precio (58\%); seguida por la calidad (32,3\%); el origen (6,5\%); y por último la apariencia $(3,2 \%)$.

Opiniones del actual sistema de alimentación escolar. En esta categoría el $19,35 \%$ de los entrevistados se declaró indiferente, mientras que el $54,83 \%$ indicó que era buena. Dentro de estos, el 29,03\% señaló como posible mejora aumentar las porciones y la cantidad de verduras incluidas en ellas. Del porcentaje restante, las opiniones negativas fueron: «ración pequeña» (12,9\%); "problemas en la repartición de raciones» $(9,67 \%)$; "falta de etiquetado sobre el origen de los productos» (3,22\%).

Opiniones de la nueva propuesta. El 67,74\% de los entrevistados omitió este ítem, mientras que el 32,25\% indicó estar a favor, señalando como argumentos que era una iniciativa saludable, otorgaría alimentos más frescos, apoyaría la agricultura y que entregaba mayor seguridad en el consumo.

Participación en el Programa. La totalidad de entrevistados considera la propuesta como una herramienta para el desarrollo de la comunidad, el 93,54\% considera que los alimentos locales son más saludables; y el $96,77 \%$ estaría dispuesto a participar en el modelo. De hecho, el 74,19\% declaraba que pagaría una cantidad adicional porque la iniciativa estuviera en marcha (Tabla 2).

Tabla 2. Disposición a pagar por nuevo modelo Table 2. Willingness to pay for new model.

\begin{tabular}{cc}
\hline $\begin{array}{c}\text { Rangos de pago } \\
\text { diario (CLP) }\end{array}$ & $\begin{array}{c}\text { Número de } \\
\text { apoderados }\end{array}$ \\
\hline 0 & 8 \\
$100-500$ & 9 \\
$501-1000$ & 13 \\
$1100-2000$ & 1 \\
\hline
\end{tabular}

Aspectos positivos y negativos. De un total de 31 entrevistados, el $80,65 \%$ señaló aspectos positivos relativos a la iniciativa como: alimentos más sanos, frescos y de mejor calidad (61,29\%); apoyo a la agricultura de la zona (16,13\%); y entrega de alimentos a buena hora (3,23\%). Solo un $29,03 \%$ señaló aspectos negativos como: atrasos en la entrega (19,35\%); problemas de abastecimiento $(6,45 \%)$; y poca o nula supervisión (3,23\%).

Exigencias. En este ítem de un total de 31 entrevistados, $30 \quad(96,77 \%)$ señalaron exigencias para la implementación, tales como: control, fiscalización y entrega a tiempo $(45,16 \%)$; calidad, nutrición y alimentación saludable (41,93\%); y porción más abundante $(9,68 \%)$.

Entrevista a responsables de cocina en comedores escolares

Como ya fue señalado, estas entrevistas se llevaron a cabo en tres establecimientos educacionales. Los resultados se detallan a continuación.

Funcionamiento. Los tres establecimientos presentaban completa equipación de la cocina, además de manejar un presupuesto y menú determinado por JUNAEB. En cuanto a las normas de calidad y sanidad se rigen por las impuestas por el Ministerio de Salud y JUNAEB.

Participación en el programa. La totalidad de los establecimientos señalaron su disposición a participar, apoyar esta iniciativa como una herramienta de impulso local, recibir fiscalización y manifestaron confianza frente a los alimentos locales.

Aspectos positivos $y$ negativos. LOS responsables de comedores de las tres entidades señalaron como características positivas de la iniciativa el tener "alimentos más frescos, sanos y de calidad»; «impulso a 
la agricultura de la zona»; "cercanía a los centros abastecedores de alimentos" y «mayor coordinación de los procesos y entregas». Como aspecto negativo una de las cocinas manifestó el posible atraso en las entregas de alimentos.

Exigencias. Estas consistieron en la fiscalización y vigilancia de la cadena productiva, el cumplimiento de entregas y normas sanitarias correspondientes.

\section{Análisis FODA}

Finalmente, con toda la información recopilada mediante los PAE brasileño y chileno y los resultados de las entrevistas, junto con el apoyo de expertos en el área de agronegocios y políticas públicas, se procedió a realizar un análisis FODA (Fortalezas, Oportunidades, Debilidades y Amenazas) relativo a la propuesta de incorporar las compras a la AFC en la alimentación escolar en Chile.

Fortalezas. Chile cuenta con un sistema de alimentación escolar coordinado por JUNAEB en funcionamiento, por lo que se tiene experiencia en el área y se cuenta con recursos logísticos. Además, los establecimientos educacionales cuentan con las estructuras y conocimientos adecuados en la manipulación de alimentos para entregar la alimentación.

En el área de producción agrícola de pequeña escala, también existe una institución, INDAP, que trabaja con este sector, por lo que se puede contar con contactos y experiencia en el trato con pequeños agricultores. Por otro lado, este sistema permite una mayor estabilidad económica al pequeño productor, al asegurar la venta de producción, generando nuevas oportunidades de ingresos $y$, en caso de incluir asesoría, el aumento de la productividad del mismo. Además, existe la posibilidad de contar con un sistema de compra sin licitación, lo que incentivaría a los productores a participar de la iniciativa.

En general, este sistema podría permitir mejorar la alimentación escolar dado que aumentarían las exigencias de calidad por parte de la población, ya que al recibir alimentos más frescos, producidos en la zona, se genera un cierto sentido de identidad y cercanía de los consumidores con los agricultores.

Debilidades. Las principales debilidades al implementar el modelo que se plantea son las posibles fallas en el abastecimiento por problemas en la producción y/o en el cumplimiento de las cuotas, así como la poca variedad de productos. Por otro lado se encuentra la dificultad de gestión por parte de los agricultores para cumplir las normas de calidad. Adicionalmente, se debe considerar la posible oposición de las concesionarias al cambio del sistema, así como la eventual demora y costos adicionales de coordinación y financiamiento, entre otros.

Oportunidades. Existen proyectos en el extranjero con buenos resultados, lo que puede servir de guía a la hora de diseñar e implementar la iniciativa, al contar con la experiencia de terceros en el tema. Por el lado de los actores involucrados en la cadena se puede establecer, según las entrevistas, que existiría una buena disposición a priori de participar del proyecto. A esto se suma la vigencia de campañas educativas para incentivar el consumo de alimentos sanos y una mayor organización y experiencia para elevar solicitudes relevantes para el sector (ej. proyectos de riego, asesorías técnicas...) por parte de los agricultores.

Amenazas. Existe competencia directa con ferias y otros canales de comercialización donde los productores pueden vender sus productos, lo que podría dificultar el abastecimiento. A su vez, podría generarse una preferencia por otras iniciativas que potencien la agricultura local, además del 
inminente poco interés en la iniciativa una vez puesta en marcha a nivel nacional, producto de la baja preocupación por la procedencia de los alimentos. Por el lado gubernamental es posible una carencia de intención en realizar los cambios institucionales eventualmente necesarios para implementar el nuevo sistema.

Este análisis permite inferir, que en el área agrícola, se deben examinar las opciones de cultivos $y / o$ rotaciones en las zonas implicadas, para generar una mayor variedad y estabilidad en la producción; mantener la ayuda mediante asesorías técnicas y financieras ya existentes (INDAP) y explorar su ampliación o posibilidad de generar mecanismos adicionales. De la misma forma, se debe considerar la implementación de tecnologías, como invernaderos, a una escala que la AFC pueda manejar y cuya ejecución sea económicamente viable. Por otro lado, políticas de precio justo son necesarias para lograr la fidelización y fortalecimiento del sector productivo, así como también disminuir la competencia por la compra de alimentos desde la AFC por otros sectores.

En cuanto a la aceptación por parte de los implicados, se debe buscar apoyo en las campañas educativas existentes acerca de la alimentación sana y adaptarlas de acuerdo con las características del modelo (identidad con la comunidad, desarrollo local, entre otros) de tal forma que mantengan y generar interés.

\section{CONCLUSIONES}

Chile presenta un gran potencial para la implementación de un modelo de compras públicas desde la agricultura familiar campesina, para el consumo social en establecimientos educacionales.
En primer lugar, se cuenta con la existencia actual de un sistema de alimentación, y con ello, las directrices fundamentales $y$ experiencia para su correcto funcionamiento. En el modelo presente es posible establecer criterios sociales en la selección de las concesionarias actualmente encargadas del abastecimiento de las escuelas, tales como la incorporación de un porcentaje mínimo de compras de materias primas desde la pequeña agricultura.

Por otro lado, se encuentra la pequeña agricultura, que con los mecanismos de incentivo como financiamiento, asesorías y seguridad en los contratos haría viable la conformación del eslabón productivo del sistema. Sin embargo, es necesaria la presencia de una institución encargada de otorgar las líneas de financiamiento $y$ asesorías, así como también el control y fiscalización de las prácticas utilizadas procurando alimentos con la regularidad, inocuidad y calidad requerida.

El sistema de alimentación actual cuenta con un marco regulador en el funcionamiento de los contratos, operaciones y aspectos sanitarios. Para la implementación de la nueva iniciativa es necesaria la ampliación de ese marco legal, incluyendo: los nuevos criterios de compras en el caso de las concesionarias; condiciones de contrato como la oportunidad en el pago y la determinación de precios, entre otras bases generales, para generar los resguardos básicos para cada uno de los participantes en el aspecto de la adquisición de alimentos; la introducción de parámetros de calidad, así como la generación de medidas de control y fiscalización en los alimentos al sector productivo; y la retroalimentación constante sobre las actividades y funcionamiento de cada parte involucrada en el modelo. 


\section{REFERENCIAS}

Banco Mundial (2008). World development report 2008: Agriculture for development. DOI: 10.1596/978-0-82137235-7

Cervato-Mancuso A., Morales G. y Pava, A. (2013) Grupo focal con nutricionistas que actúan en la alimentación escolar: el menú como elemento pedagógico. Revista chilena de nutrición, 40(3), 250255.

Compañía Nacional de Abastecimiento Gobierno de Brasil (2005). Apoio à comercialização da agricultura familiar: Programa de Aquisição de Alimentos. Recuperado de: http://www.fnde.gov.br/index.php/aeencontros-tecnicos

Da Cunha, D., Assunção R., Ribeiro, R., De Lacerda, L. y Stedefeldt, E. (2013). Métodos para aplicar las pruebas de aceptación para la alimentación escolar: validación de la tarjeta lúdica. Revista chilena de nutrición, 40(4), 357-363.

Dirección de Presupuestos Gobierno de Chile (2012). Balance de Gestión Integral Año 2012: Junta Nacional de Auxilio Escolar y Becas. Chile: DIPRES.

Figueroa, D. y Lucema S. (2005). La alimentación escolar analizada en el contexto de un programa. Revista Costarricense de salud pública, 14(26), 28-29.

Fondo Nacional para el Desarrollo de la Educación Gobierno de Brasil (2012). Programa de Alimentación Escolar. Recuperado de: http://www.fnde.gov.br/index.php/prog ramas-alimentacao-escolar

Instituto Interamericano de Cooperación para la Agricultura (2007). La agricultura familiar en los países del Cono Sur. Asunción: IICA.
Instituto de Desarrollo Agropecuario Gobierno de Chile (2008). Normas técnicas y procedimentos operativos del Programa de Desarrollo Local, PRODESAL. Santiago: INDAP. 56p.

Instituto de Desarrollo Agropecuario Gobierno de Chile (2009). Marco Programa de Desarrollo Local. Santiago: INDAP.

Instituto de Desarrollo Agropecuario Gobierno de Chile (2010a). Caracterización de usuarios y rubros. Recuperado:

http://www.indap.gob.cl/Prodesal/Pagi nas/Caracterizaci\%c3\%b3nUsuariosyRub ros.aspx

Instituto de Desarrollo Agropecuario Gobierno de Chile (2010b). Financiamiento del Programa de Desarrollo Local. Recuperado de 2012 de:

http://www.indap.gob.cl/Prodesal/Pagi nas/Presupuesto.aspx

Instituto Nacional de Estadísticas Gobierno de Chile (2007). VII Censo Agrpecuario y Forestal 2007: Superficie cultivada con hortalizas, año agrícola 2006/2007, por sistema de cultivo, según región, provincia y especie. Recuperado de: http://www.ine.cl/canales/chile_estadis tico/censos_agropecuarios/censo_agrop ecuario_07_comunas.php

Junta Nacional de Auxilio Escolar y Becas Gobierno de Chile (2008). Bases Técnicas y Operativas Programa de Alimentación Escolar, Licitación PAE 25/2008. Chile: JUNAEB.

Junta Nacional de Auxilio Escolar y Becas Gobierno de Chile (2014). Programa Alimentación Escolar. Recuperado de: http://www.junaeb.cl/programa-dealimentacion-escolar

Márcia R. (2010). Reconectando a produção ao consumo: a aquisição de gêneros alimentícios da agricultura familiar para 
- Programa de Alimentação Escolar. Universidade Federal Do Rio Grande Do Sul. Porto Alegre, Brasil.

Ministerio de Desarrollo Agrario Gobierno de Brasil (2012). FOME ZERO (Programa Hambre Cero): La Experiencia Brasileña. Brasilia: MDA. 388p

Ministerio de Desarrollo Agrario Gobierno de Brasil (2014). Programas: Crédito Rural. Recuperado de: http://portal.mda.gov.br/portal/saf/pro gramas/pronaf

Organización de las Naciones Unidas para la Alimentación y la Agricultura (2006). Brasil, Hambre Cero: Principales Lecciones. Santiago: FAO.

Organización de las Naciones Unidas para la Agricultura y la Alimentación, Banco Interamericano de Desarrollo (2007). Políticas para la agricultura familiar en América Latina y el Caribe. Recuperado de:

http://www.fao.org/fileadmin/user_upl oad/AGRO_Noticias/docs/politicasafres u.pdf
Organización de las Naciones Unidas para la Alimentación y la Agricultura (2013). Alimentación escolar y las posibilidades de compra directa de la agricultura familiar: Estudio de caso de ocho países. Recuperado de: http://www.fao.org/docrep/018/i3413s /i3413s.pdf

Programa de las Naciones Unidas para el Medio Ambiente (2012). Implementando compras públicas sostenibles. Introducción al enfoque de PNUMA. Recuperado de: http://www.unep.org/resourceefficienc y/Portals/24147/scp/10yfp/document/S PPguidelines_SP_27.07.12.pdf

Pontificia Universidad Católica de Chile (2010). Evaluación de Impacto de los Programas de INDAP: Programa de Desarrollo Local - Programa de Desarrollo de Comunas Pobres. Santiago: PUC. 